\title{
Variation in Virulence \\ Within Meloidogyne chitwoodi, M. fallax, and M. hapla on Solanum spp.
}

\author{
J. G. van der Beek, L. M. Poleij, C. Zijlstra, R. Janssen, and G. J. W. Janssen
}

First, second, and third authors: Research Institute for Plant Protection (IPO-DLO), P.O. Box 9060, NL-6700 GW Wageningen, the Netherlands; fourth and fifth authors: Centre for Plant Breeding and Reproduction Research (CPRO-DLO), P.O. Box 16, NL-6700 AA Wageningen, the Netherlands.

Accepted for publication 19 March 1998.

\begin{abstract}
Van der Beek, J. G., Poleij, L. M., Zijlstra, C., Janssen, R., and Janssen, G. J. W. 1998. Variation in virulence within Meloidogyne chitwoodi, M. fallax, and M. hapla on Solanum spp. Phytopathology 88:658-665.

The virulence of Meloidogyne hapla, M. chitwoodi, and M. fallax was studied on genotypes of Solanum spp. in a greenhouse. Juveniles of $11 M$. hapla race A isolates, $3 \mathrm{M}$. hapla race $\mathrm{B}$ isolates, and 5 mono-female lines of a $M$. hapla race A isolate were inoculated on $S$. chacoense, $S$. hougasii, and $S$. sparsipilum. Juveniles of eight $M$. chitwoodi isolates, five $M$. fallax isolates, and six mono-female lines of a $M$. chitwoodi isolate were inoculated on S. bulbocastanum, S. chacoense, S. hougasii, S. stoloniferum, and S. tuberosum. Virulence was expressed as nematode reproduction 8 weeks after inoculation. Nematode reproduction was estimated by the number of egg masses and, in one experiment, by the number of hatched second-stage juveniles per inoculated juvenile. Considerable variation in virulence and resistance was observed among $M$.
\end{abstract}

ABSTRACT

The root-knot nematode species Meloidogyne chitwoodi Golden et al. and M. hapla Chitwood can cause growth and yield reductions and quality loss in a large number of crops. In potato (Solanum tuberosum L.), these root-knot nematodes are able to reproduce on roots, causing yield reduction (21). M. fallax Karssen, which was described recently (22), is allopatric with $M$. chitwoodi in the Netherlands, and its host range is similar to that of $M$. chitwoodi. M. hapla causes little quality damage due to gall formation on the outside of tubers, in contrast to M. chitwoodi (7). Recent observations indicate that $M$. chitwoodi and M. fallax reproduce similarly on tubers, and both result in quality loss (J. G. van der Beek, unpublished data). In addition to yield reduction and quality loss, an increase in the nematode population due to cultivation of a susceptible host makes these pathogens a potential threat to agriculture in Western Europe, because many crops in the current rotation schemes are good hosts (33). However, M. hapla does not reproduce on monocotyledonous crops, which may be used in rotation with dicotyledons as an effective control measure.

The introduction of resistant potato cultivars also would improve the efficiency of crop rotation in reducing this threat. However, in current potato cultivars, no desirable level of resistance to $M$. chitwoodi and $M$. hapla has been identified $(7,17)$.

Thus far, intraspecific variation in $M$. chitwoodi has been described by the occurrence of three host races that can be distinguished on alfalfa cv. Thor (nonhost for race 1 and host for races 2 and 3), carrot cv. Red Cored Chantenay (host for race 1 and non-

Corresponding author: J. G. van der Beek

E-mail address: j.g.vanderbeek@IPO.DLO.NL

Publication no. P-1998-0427-01R

(C) 1998 The American Phytopathological Society hapla isolates and plant genotypes, respectively. The M. hapla isolateplant species interaction was highly significant. The response to $M$. chitwoodi ranged from susceptible ( $S$. tuberosum and $S$. chacoense) to highly resistant (S. bulbocastanum and S. hougasii). S. tuberosum was susceptible to $M$. fallax, whereas all four wild species were resistant. In contrast to $M$. hapla, no significant isolate-plant genotype interaction was obtained for $M$. chitwoodi or $M$. fallax, indicating no or little intraspecific variation in virulence. $M$. chitwoodi juveniles in species mixtures with $M$. fallax isolates appeared to be able to break the resistance of $S$. bulbocastanum and S. hougasii. Significant differences among mono-female lines of $M$. hapla and $M$. chitwoodi were observed, indicating heterogeneity of pathogenicity within meiotic parthenogenic Meloidogyne populations.

Additional keywords: genetic variation, intraspecific specialization, potato, root-knot nematode. host for races 2 and 3), and S. bulbocastanum Dun. SB22 (nonhost for races 1 and 2 and host for race 3$)(26,27,36)$.

Resistance to $M$. chitwoodi and $M$. fallax has been identified in accessions of wild tuber-bearing Solanum species, originating mainly from Central America, such as S. bulbocastanum and S. hougasii Corr. $(5,6,18)$. Resistance to $M$. chitwoodi has been transferred from a resistant genotype of $S$. bulbocastanum to the gene pool of the cultivated potato by somatic hybridization $(1,7)$, and a single gene has been localized on chromosome 11 that appears to control this resistance (8). Also, various levels of resistance to M. hapla have been reported $(9,15,18)$. Recent investigations indicated the presence of variation in host responses to different $M$. hapla isolates on some genotypes of $S$. bulbocastanum, S. hougasii, $S$. chacoense Bitt., S. gourlayi, S. sparsipilum, and S. spegazzinii that were selected for resistance (19).

In general, the efficacy of resistance depends largely on genetic variation in the virulence of the pathogen and factors involved in the durability of resistance. Often the presence of resistance is concluded from experiments with one or a few isolates. The current study focused on the variation in virulence within $M$. chitwoodi, M. fallax, and M. hapla by testing several isolates and mono-female lines on some completely resistant and incompletely resistant genotypes of Solanum spp. In this paper, virulence is defined as the ability of an isolate of a Meloidogyne species to reproduce significantly on resistant host plants that prevent or suppress reproduction of other isolates of the Meloidogyne species (adapted from Roberts [34]).

\section{MATERIALS AND METHODS}

Nematode isolates and plant genotypes. A total of $8 \mathrm{M}$. chitwoodi, $5 \mathrm{M}$. fallax, and $14 \mathrm{M}$. hapla isolates were used in this 
study (Table 1). All isolates, except three, were from geographically different sites in the Netherlands, and originated from various hosts. M. chitwoodi isolate Cba was obtained from the United States (Oregon) and represents race 1; M. hapla isolates Haw and Han originated from France and South Korea, respectively. Of the $M$. hapla isolates, 11 were of the facultative meiotic parthenogenetic race $\mathrm{A}$, and 3 were of the obligate mitotic parthenogenetic race $\mathrm{B}$. Races $\mathrm{A}$ and $\mathrm{B}$ were distinguished by examining oocytes for the presence (race A) or absence (race B) of a second maturation division, using Hoechst 33258 (Polysciences Inc., Warrington, PA) for chromosome staining (42). All isolates were maintained and propagated on Lycopersicon esculentum L. 'Moneymaker' in glass-house compartments at $20 \pm 2{ }^{\circ} \mathrm{C}$ and 70 to $80 \%$ relative humidity (RH). During maintenance, the isolates were tested regularly for possible contamination with other species by observing isozyme phenotypes of esterase and malate dehydrogenase $(12,23)$ and analysis of internal transcribed spacer (ITS) regions of ribosomal DNA $(46,47)$. Additionally, during the experiment, samples of the inocula of the isolates were collected and verified for absence of contamination by analysis of ITS regions. The same DNA test was performed with samples of nematode progenies of the isolates extracted from test plants at harvest.

Six mono-female lines (Cb1 to Cb6) from M. chitwoodi isolate $\mathrm{Cb}$ and five mono-female lines (Ha1, $\mathrm{Ha} 2, \mathrm{Ha} 4, \mathrm{Ha} 5$, and $\mathrm{Ha} 6$ ) from $M$. hapla race A isolate Ha were developed by inoculating root tips of $S$. tuberosum cv. Eigenheimer grown in petri dishes containing 2.0 technical agar no. 3 (Oxoid, London) with secondstage juveniles (J2). To ensure parthenogenetic offspring, only one $\mathrm{J} 2$ was inoculated per petri dish. Consequently, the progeny of such a juvenile are termed a mono-female line. This procedure was repeated for seven consecutive cycles in petri dishes. The mono-female lines obtained each originated from a different egg mass of the original isolates, with one exception: $\mathrm{Cb} 1$ and $\mathrm{Cb} 2$ were derived from two $\mathrm{J} 2$ from the same egg mass after one generation of parthenogenesis. The resulting 11 single egg masses were propagated separately on plants of cv. Moneymaker prior to preservation in liquid nitrogen (43). After thawing, two generations followed on tomato plants to obtain adequate amounts of $\mathrm{J} 2$ for the experiment.

The nematode isolates and mono-female lines of M. chitwoodi and $M$. fallax were tested on susceptible $S$. tuberosum cv. Nicola and genotypes of four wild Solanum spp.: S. bulbocastanum 9360-2, S. chacoense 93-68-2, S. hougasii 93-71-6, and S. stoloniferum 93-STOL-2. The nematode isolates and mono-female lines of $M$. hapla were tested on genotypes of three wild tuber-bearing Solanum spp.: S. chacoense 93-68-2, S. hougasii 93-71-6, and S. sparsipilum 93-107-1. Genotypes of the wild species were selected from previous experiments because of their observed variation in response to one, two, or three isolates of M. chitwoodi, $M$. fallax, or M. hapla (19). In those experiments, the S. bulbocastanum and $S$. hougasii genotypes were completely resistant to $M$. chitwoodi and $M$. fallax, $S$. stoloniferum was incompletely resistant to $M$. chitwoodi and completely resistant to $M$. fallax, and $S$. chacoense was susceptible to $M$. chitwoodi and completely resistant to $M$. fallax. Except for $S$. tuberosum cv. Nicola, these genotypes were maintained and propagated in vitro, ensuring rapid multiplication. To obtain the proper number of plants per genotype, shoots were cut every 2 to 3 weeks and transferred to new tubes with Murashige and Skoog medium containing $30 \mathrm{~g}$ of sucrose per liter (28). Three weeks after the last cut, plantlets were large enough for in vivo transplantation.

Test for virulence. In vitro plantlets of the wild Solanum spp. and tuber pieces with top sprouts of $\mathrm{cv}$. Nicola were transplanted to 350-ml clay pots, which were filled with moist silver sand containing $0.2 \%$ slow-release fertilizer (Osmocote; Sierra Chemical Company, Milpitas, CA) and $0.08 \%$ N-P-K (12-10-18) and placed in a greenhouse compartment at $20 \pm 2^{\circ} \mathrm{C}$ at 70 to $80 \% \mathrm{RH}$ with additional light for $16 \mathrm{~h}$ per day. During the first 4 days after transplantation, $\mathrm{RH}$ was kept at $\approx 100 \%$ by covering the plants originating from in vitro culture with transparent plastic.

To prepare the nematode inoculum, heavily infested tomato roots were placed on trays in a mist cabinet at $20^{\circ} \mathrm{C}$ with a water temperature of $25^{\circ} \mathrm{C}$ (37). One- to two-day-old hatched $\mathrm{J} 2$, which settled on the bottom of the tray, were collected over approximately three weeks and kept at $4{ }^{\circ} \mathrm{C}$. Prior to inoculation, the $\mathrm{J} 2$ were poured over a paper filter. Inoculum was collected after 1 day, and inoculation was done on plantlets randomly assigned to treatment by twice applying a quantity of $4.5 \mathrm{ml}$ of inoculum in a groove $\approx 1 \mathrm{~cm}$ deep around the plant while continuously mixing the inoculum. An average inoculation density of $1.6 \mathrm{~J} 2$ per $\mathrm{ml}$ of sand was used, resulting in a total of $\approx 560 \mathrm{~J} 2$ per pot. Noninoculated control plants were included in the experiment.

For practical reasons, tests with $M$. chitwoodi and $M$. fallax isolates were carried out at a different time than tests with $M$. hapla. Also, tests with the mono-female lines were carried out at a different time than those with the isolates. This resulted in four experiments arranged in a complete randomized block design, each with five replicates. Eight weeks after inoculation, the roots were rinsed free of sand and stained with phloxine B (10). Egg masses were counted visually.

For the experiments with $M$. chitwoodi and M. fallax isolates and lines, roots were ground in a table blender, and eggs were extracted by shaking the roots for $4 \mathrm{~min}$ in $0.5 \% \mathrm{NaOCl}$ (extraction method adapted from Hussey and Barker [16]). Eggs were

TABLE 1. Meloidogyne hapla, M. chitwoodi, and M. fallax isolates used in the experiments

\begin{tabular}{|c|c|c|c|c|}
\hline Isolate & Origin $^{x}$ & Host of origin & $\begin{array}{c}\text { Year of } \\
\text { sampling }\end{array}$ & Obtained $^{\mathrm{y}}$ \\
\hline \multicolumn{5}{|l|}{ M. hapla ${ }^{z}$} \\
\hline На (A) & Lisse & Peony & 1990 & PD \\
\hline $\mathrm{Hb}(\mathrm{A})$ & Zwaanshoek & Astilbe sp. & 1990 & PD \\
\hline $\mathrm{Hc}(\mathrm{A})$ & Bavel & Aconitum sp. & 1990 & PD \\
\hline $\mathrm{He}(\mathrm{A})$ & Drouwenerveen & Potato & 1992 & PAV \\
\hline $\mathrm{Hf}(\mathrm{A})$ & Borgers & Carrot & 1992 & PAV \\
\hline $\mathrm{Hi}(\mathrm{A})$ & Smilde & Immortelle & 1992 & PAV \\
\hline $\mathrm{Hj}(\mathrm{A})$ & Smilde & Carrot & 1992 & PAV \\
\hline Hk (A) & Slochteren & Chicory & 1992 & PAV \\
\hline Hak (A) & Mussel & Carrot & 1993 & HLB \\
\hline Haw (A) & Antibes (Fr.) & Grape & Pre-1977 & INRA \\
\hline Hbz (A) & Amerzoden & Rose & 1990 & PD \\
\hline $\mathrm{Hh}(\mathrm{B})$ & Wachtum & Fallow (weed) & 1992 & PAV \\
\hline Han (B) & S. Korea & Hosta sp. & 1990 & PD \\
\hline Hbr (B) & Hoorn & Veronica sp. & 1992 & PD \\
\hline \multicolumn{5}{|c|}{ M. chitwoodi } \\
\hline $\mathrm{Ca}$ & NL & Maize & 1989 & PD \\
\hline $\mathrm{Cb}$ & NL & Wheat & 1990 & PD \\
\hline $\mathrm{Ck}$ & NL & Tomato & 1989 & PD \\
\hline Co & NL & Black salsify & 1993 & PAV \\
\hline $\mathrm{Cx}$ & NL & Potato & 1993 & PD \\
\hline Су & NL & Potato & 1993 & PD \\
\hline $\mathrm{Caq}$ & NL & Potato & 1993 & CPRO \\
\hline $\mathrm{Cba}$ & OR & Potato & Unknown & WSU \\
\hline \multicolumn{5}{|l|}{ M. fallax } \\
\hline $\mathrm{Fa}$ & NL & Early primrose & 1992 & PAGV \\
\hline $\mathrm{Fb}$ & NL & Beet & 1991 & PD \\
\hline $\mathrm{Fc}$ & NL & Potato & 1993 & PD \\
\hline $\mathrm{Fd}$ & NL & Potato & 1993 & PD \\
\hline $\mathrm{Fe}$ & NL & Unknown & 1994 & BLGG \\
\hline
\end{tabular}

${ }^{\mathrm{x}}$ Fr. $=$ France; $\mathrm{NL}=$ Netherlands; Oregon $=$ Oregon .

${ }^{y} \mathrm{PD}=$ Plant Protection Service, Wageningen, the Netherlands; PAV $=$ Research Station for Arable Farming and Field Production of Vegetables, Lelystad, the Netherlands; HLB = Hilbrands Laboratory for Soilborne Diseases and Pests, Assen, the Netherlands; INRA = Institut National de Recherche Agronomique, Montpellier, France; CPRO = DLO-Centre for Plant Breeding and Reproduction Research, Wageningen, the Netherlands; WSU = Washington State University, Department of Plant Pathology, Prosser; BLGG = Laboratory for Soil and Crop Testing, Oosterbeek, the Netherlands.

${ }^{\mathrm{z}}$ Letters in parentheses indicate cytological race. 
separated from root particles with a series of sieves. Subsequently, eggs were collected and hatched on $20-\mu \mathrm{m}$ mesh sieves, and J2 were counted after 3 weeks of incubation at $20^{\circ} \mathrm{C}$. Total numbers of hatched $\mathrm{J} 2$ were estimated by counting three samples of $1 \mathrm{ml}$ of J2 suspension with a Contextvision MicroGOP2000 image analysis package (Struers Vision AB, Linköping, Sweden) (3). The reproduction rate, $P f / P i$ (final population divided by initial population), was estimated by the number of hatched $\mathrm{J} 2$ per inoculated J2. The root volume was estimated according to a scale with four response categories ranging from small $(=1)$ to large $(=4)$ root volume.

Statistical analysis. Linear logistic regression was employed to explain the number of egg masses observed $(Y)$ in response to

TABLE 2. Mean number of egg masses for combinations of Meloidogyne hapla isolates and Solanum chacoense, S. hougasii, and S. sparsipilum

\begin{tabular}{lcccr}
\hline & \multicolumn{3}{c}{ Host $^{\mathrm{z}}$} & $\begin{array}{c}\text { Isolate } \\
\text { mean }\end{array}$ \\
\cline { 2 - 4 } Isolate $^{\mathrm{y}}$ & S. chacoense & S. hougasii & S. sparsipilum & \\
\hline $\mathrm{Ha}$ & $0.5 \mathrm{a}$ & $8.8 \mathrm{~b}$ & $0.0 \mathrm{a}$ & 3.1 \\
$\mathrm{Hb}$ & $0.0 \mathrm{a}$ & $2.0 \mathrm{a}$ & $0.0 \mathrm{a}$ & 0.6 \\
$\mathrm{Hc}$ & $0.0 \mathrm{a}$ & $3.8 \mathrm{~b}$ & $1.2 \mathrm{ab}$ & 1.6 \\
$\mathrm{He}$ & $1.8 \mathrm{a}$ & $16.4 \mathrm{~b}$ & $4.5 \mathrm{a}$ & 7.6 \\
$\mathrm{Hf}$ & $28.5 \mathrm{~b}$ & $0.0 \mathrm{a}$ & $48.5 \mathrm{c}$ & 25.6 \\
$\mathrm{Hi}$ & $8.3 \mathrm{a}$ & $35.3 \mathrm{~b}$ & $41.0 \mathrm{~b}$ & 28.2 \\
$\mathrm{Hj}$ & $6.0 \mathrm{~b}$ & $0.0 \mathrm{a}$ & $41.8 \mathrm{c}$ & 15.9 \\
$\mathrm{Hk}$ & $0.3 \mathrm{a}$ & $0.0 \mathrm{a}$ & $3.0 \mathrm{a}$ & 1.0 \\
$\mathrm{Hak}$ & $10.4 \mathrm{~b}$ & $0.0 \mathrm{a}$ & $43.8 \mathrm{c}$ & 18.1 \\
$\mathrm{Haw}$ & 0.8 & 0.0 & 15.8 & 5.5 \\
$\mathrm{Hbz}$ & $14.5 \mathrm{~b}$ & $0.4 \mathrm{a}$ & $2.8 \mathrm{a}$ & 5.9 \\
$\mathrm{Hh}$ & $3.3 \mathrm{a}$ & $2.2 \mathrm{a}$ & $1.5 \mathrm{a}$ & 2.3 \\
$\mathrm{Han}$ & 11.4 & 0.0 & 3.8 & 5.1 \\
$\mathrm{Hbr}$ & $0.6 \mathrm{a}$ & $2.2 \mathrm{a}$ & $4.0 \mathrm{a}$ & 2.3 \\
& & & & 8.5 \\
Host mean & 6.0 & 4.6 & 14.7 & 8.7 \\
\hline
\end{tabular}

y Isolates Haw and Han were contaminated with M. chitwoodi and excluded from the statistical analysis.

${ }^{\mathrm{z}}$ Means with common letters in a row are not significantly different at $P<$ 0.05 , using the $F$ distribution for the deviance statistic on the logit scale.

TABLE 3. Mean number of egg masses for combinations of Meloidogyne hapla isolate $\mathrm{Ha}$ and mono-female lines of isolate Ha with Solanum chacoense, S. hougasii, and S. sparsipilum

\begin{tabular}{|c|c|c|c|c|}
\hline \multirow[b]{2}{*}{ Line } & \multicolumn{3}{|c|}{ Host } & \multirow{2}{*}{$\begin{array}{l}\text { Isolate } \\
\text { mean }^{\mathrm{z}}\end{array}$} \\
\hline & S. chacoense & S. hougasii & S. sparsipilum & \\
\hline $\mathrm{Ha}$ & 0.2 & 13.8 & 0.4 & $4.8 \mathrm{a}$ \\
\hline Ha1 & 0.8 & 38.7 & 0.6 & $13.4 \mathrm{~b}$ \\
\hline $\mathrm{Ha} 2$ & 0.0 & 13.6 & 0.6 & $4.7 \mathrm{a}$ \\
\hline $\mathrm{Ha} 4$ & 0.0 & 3.0 & 0.2 & $1.1 \mathrm{a}$ \\
\hline $\mathrm{Ha} 5$ & 0.0 & 12.0 & 0.0 & $4.0 \mathrm{a}$ \\
\hline Ha6 & 0.8 & 41.0 & 1.2 & $14.3 \mathrm{~b}$ \\
\hline Host mean ${ }^{z}$ & $0.3 \mathrm{p}$ & $22.0 \mathrm{q}$ & $0.5 \mathrm{p}$ & \\
\hline
\end{tabular}

${ }^{\mathrm{z}}$ Means followed by the same letter are not significantly different at $P<0.05$, using the $F$ distribution for the deviance statistic on the logit scale.

TABLE 4. Analysis of deviance for linear logistic regression of egg-mass counts of Meloidogyne hapla isolates inoculated on Solanum chacoense, S. hougasii, and $S$. sparsipilum

\begin{tabular}{lrrrr}
\hline Source & df & Deviance & $\begin{array}{c}\text { Mean } \\
\text { deviance }\end{array}$ & $\begin{array}{c}\text { Deviance } \\
\text { ratio }^{\mathrm{z}}\end{array}$ \\
\hline $\begin{array}{l}\text { Replication } \\
\text { Isolates }\end{array}$ & 4 & 47.4 & 11.84 & $2.55^{*}$ \\
$\begin{array}{l}\text { Solanum genotype } \\
\text { Isolates-Solanum }\end{array}$ & 2 & $1,451.5$ & 131.95 & $28.39^{* * *}$ \\
$\quad \begin{array}{l}\text { genotype } \\
\text { Residual }\end{array}$ & 22 & $1,016.3$ & 46.20 & $9.94^{* * *}$ \\
Total & 122 & 567.1 & 4.65 & \\
\hline
\end{tabular}

$\mathrm{z} *$ and $* * *$ indicate deviance ratios are significant at $P<0.05$ and $P<0.001$, respectively, using the $F$ distribution. several classifying variables: replicate, Solanum genotype, Meloidogyne isolate, and interaction between Solanum genotype and Meloidogyne isolate. The model assumes that the variance in the number of egg masses observed is due to a distribution more variable than the binomial and that the variance can be adequately described by variance proportional to binomial variance (24). The linear logistic model is represented as

$$
\begin{aligned}
& \log \left[\mu_{i j k} /\left(n-\mu_{i j k}\right)\right]=\text { constant }+ \text { replicate }_{i}+\text { isolate }_{j}+\text { genotype }_{k}+ \\
& \text { isolate } \cdot \text { genotype }_{j k} \\
& \operatorname{var}\left(Y_{i j k}\right)=\sigma^{2} \mu_{i j k}\left(n-\mu_{i j k}\right) / n
\end{aligned}
$$

where $Y_{i j k}$ and $\mu_{i j k}$ are the observed and expected numbers of egg masses for isolate $j$ and genotype $k$ in replicate $i$, respectively, $n$ is the number of inoculated $\mathrm{J} 2$ and denotes the dispersion parameter. If $\sigma^{2}>1$, there is overdispersion compared to binomial variance.

Loglinear regression models with variance proportional to Poisson variance were employed to describe the response of the ratios of the number of produced $\mathbf{J} 2$ and number of inoculated $\mathbf{J} 2$ to the same classifying variables. The loglinear model is represented as

$$
\begin{aligned}
& \log \left(\mu_{i j k}\right)=\text { constant }+ \text { replicate }_{i}+\text { isolate }_{j}+\text { genotype }_{k}+\text { isolate } \cdot \text { genotype }_{j k} \\
& \operatorname{var}\left(R_{i j k}\right)=\phi \mu_{i j k}
\end{aligned}
$$

where $R_{i j k}$ and $\mu_{i j k}$ are the observed and expected values of the ratio for isolate $j$ and genotype $k$ in replicate $i$, respectively, and $\phi$ denotes the dispersion parameter. If $\phi>1$, there is overdispersion compared to Poisson variance.

The logistic and loglinear models were fitted to the data by the quasi-likelihood method (45). For the logistic and loglinear models, analysis of deviance was used as a generalization of the analysis of variance (ANOVA) for data with normal errors. In an analysis of deviance table, each deviance represents the variation accounted for by its corresponding term, having eliminated the effects of terms above it but ignoring any effects of those terms below it. The criterion for inclusion of a term in the model was an $F$ test for the ratio of the mean deviance for the particular term to a current estimate for the overdispersion (the mean deviance of the rest).

TABLE 5. Analysis of deviance for linear logistic regression of egg-mass counts of Meloidogyne hapla isolate Ha and mono-female lines from isolate Ha inoculated on Solanum chacoense, S. hougasii, and S. sparsipilum

\begin{tabular}{lrcrc}
\hline Source & df & Deviance & $\begin{array}{c}\text { Mean } \\
\text { deviance }\end{array}$ & $\begin{array}{c}\text { Deviance } \\
\text { ratio }^{z}\end{array}$ \\
\hline Replication & 4 & 123.7 & 30.92 & $4.56^{* * *}$ \\
$\begin{array}{l}\text { Lines } \\
\text { Solanum genotype }\end{array}$ & 5 & 219.1 & 43.83 & $6.46^{* * *}$ \\
$\begin{array}{l}\text { Lines-Solanum } \\
\text { genotype }\end{array}$ & 10 & 954.9 & 477.45 & $70.40^{* * *}$ \\
Residual & 61 & 413.9 & 0.96 & 0.14 \\
Total & 82 & $1,721.1$ & 6.79 & \\
\hline
\end{tabular}

$\mathrm{z} * * *$ indicates deviance ratio is significant at $P<0.001$, using the $F$ distribution.

TABLE 6. Mean number of egg masses for two Meloidogyne hapla isolates (Hi and Hk) compared with two related isolates on three Solanum spp. in separate experiments

\begin{tabular}{lccc}
\hline & \multicolumn{3}{c}{ Host } \\
\cline { 2 - 4 } Isolate $^{\mathrm{z}}$ & S. chacoense & S. hougasii & S. sparsipilum \\
\hline Hi & 8.3 & 35.3 & 41.0 \\
HSMB & 32.8 & 6.0 & 21.3 \\
Hk & 0.3 & 0.0 & 3.0 \\
HSL & 0.8 & 15.3 & 3.0 \\
\hline
\end{tabular}

$\bar{z}$ The pairs of isolates Hi and HSMB and Hk and HSL originated as pairs from the same soil samples in 1992 but were propagated separate from each other for 4 years. 
Pairwise differences between treatment means on the logit scale were assessed by $t$ tests. However, in cases in which zero treatment means were obtained, $t$ tests for assessing pairwise differences between treatment means on the logistic or logarithmic scales were no longer valid. Fitting logistic and loglinear models in the case of zero means results in underflow (2), as the result of an inability to represent $-\infty$ (the estimated treatment mean on the logistic or logarithmic scale) on a computer.

Zero means were considered random, assuming that positive counts were possible. Pairwise testing was performed by an $F$ test for the ratio of the difference between residual deviances of the model, considering the groups compared as one common group, the model as different, and the residual mean deviance from the latter model. All statistical analyses were performed by Genstat 5 (release 3, Clarendon Press, Oxford).

\section{RESULTS}

M. hapla on Solanum spp. Analysis of rDNA of M. hapla juveniles hatched from eggs collected from roots 8 weeks after inoculation revealed no contamination of the isolates with other Meloidogyne spp., except for Han and Haw, which each contained $\approx 5 \%$ M. chitwoodi. Virulence data for Han and Haw, therefore, should be interpreted cautiously. These two isolates were omitted from the statistical analysis.

None of the three Solanum genotypes showed absolute resistance to all M. hapla isolates, and none of the nematode isolates showed absolute avirulence to all three Solanum genotypes (Tables 2 and 3). However, 25\% of the isolate-Solanum genotype combinations were incompatible. The plots of deviance residuals against fitted values showed no severe anomalies (data not shown). Based on calculated $P$ values for the $F$ tests of the mean deviance ratios, significant effects for the interactions between Solanum genotypes and M. hapla isolates were detected (Table 4). For each isolate, pairwise differences between the means were analyzed (Table 2). Large differences among paired means were found, for example, between isolates $\mathrm{He}$ and Hf for combinations with all three Solanum species (Table 2).

Significant effects of Solanum genotype and mono-female lines were detected, but the line-Solanum genotype interaction was not statistically significant (Table 5) in contrast to the isolate-Solanum genotype interaction (Table 4). Mean numbers of egg masses of the mono-female lines were higher than or equal to those of isolate Ha (Table 3).

The host responses of isolates $\mathrm{Hi}$ and $\mathrm{Hk}$ were compared to those of isolates from a previous study (19), HSMB and HSL, respectively. Isolates Hi and HSMB were collected in 1992 from the same soil sample from Smilde, and isolates Hk and HSL were collected from Slochteren (both sites in the Netherlands) but maintained and propagated separately. Isolates HSMB and HSL were tested by Janssen et al. (19) on the same Solanum genotypes used in this study. Large differences in virulence were found between these paired isolates (Table 6). Isolate HSL could reproduce moderately on the $S$. hougasii genotype, whereas isolate Hk was completely avirulent. Isolates HSMB and Hi varied on all genotypes with regard to virulence.

M. chitwoodi and M. fallax on Solanum spp. The tested genotypes of $S$. bulbocastanum and $S$. hougasii demonstrated extremely high levels of resistance to all $M$. chitwoodi and M. fallax isolates and mono-female lines (Tables 7 and 8). Only three isolates were able to reproduce on one or both of these two Solanum spp. Isolate Fc was able to reproduce on the S. bulbocastanum and $S$. hougasii genotypes (Table 7). Isolates $\mathrm{Fd}$ and $\mathrm{Ck}$ produced only one egg mass each on $S$. bulbocastanum and $S$. hougasii, respectively (Table 7).

Restriction analysis of ITS regions of rDNA, generated from J2 from inocula and nematode progenies extracted from cv. Nicola, indicated that these isolates $(\mathrm{T}, \mathrm{Fc}, \mathrm{Fd}$, and $\mathrm{Ck}$ ) were contaminated with one other species. $M$. chitwoodi isolate $\mathrm{Ck}$ was contaminated with $10 \%$ juveniles of $M$. hapla, which could explain the single egg mass found on $S$. hougasii. In two M. fallax isolates, low levels of contamination with $M$. chitwoodi were found: $\mathrm{Fc}$ and $\mathrm{Fd}$ both contained $\approx 2 \% M$. chitwoodi juveniles in the inocula, which resulted in $\approx 10 \%$ contamination in their progenies on cv. Nicola at 8 weeks after inoculation. The resistance-breaking ability of $M$. fallax isolate $\mathrm{Fc}$ on $S$. bulbocastanum appeared to be totally attributable to $M$. chitwoodi, which was present as a contaminant in Fc, because DNA analysis of the extracted $\mathrm{J} 2$ from $S$. bulbocastanum revealed only the $M$. chitwoodi pattern. This result

TABLE 7. Mean number of egg masses (EM) and final population divided by initial population (Pf/Pi) for combinations of Meloidogyne chitwoodi and M. fallax isolates and Solanum bulbocastanum, S. chacoense, S. hougasii, S. stoloniferum, and S. tuberosum, arranged according to increasing overall mean number of egg masses

\begin{tabular}{|c|c|c|c|c|c|c|c|c|c|c|c|c|}
\hline \multirow[b]{3}{*}{ Isolate $^{\mathrm{w}}$} & \multicolumn{10}{|c|}{ Host } & & \\
\hline & \multicolumn{2}{|c|}{ S. bulbocastanum ${ }^{\mathrm{y}}$} & \multicolumn{2}{|c|}{ S. chacoense } & \multicolumn{2}{|c|}{ S. hougasii } & \multicolumn{2}{|c|}{ S. stoloniferum } & \multicolumn{2}{|c|}{ S. tuberosum } & \multicolumn{2}{|c|}{$\operatorname{Mean}^{\mathrm{x}}$} \\
\hline & EM & $P f / P i$ & EM & $P f / P i$ & EM & $P f / P i$ & EM & $P f / P i$ & EM & $P f / P i$ & EM & $P f / P i$ \\
\hline Co & 0 & 0 & 23.8 & 13.6 & 0 & 0 & 2.4 & 2.2 & 76.4 & 24.3 & $21.4 \mathrm{a}$ & $8.6 \mathrm{a}$ \\
\hline $\mathrm{Ck}$ & 0 & 0 & 33.6 & 12.3 & 0.3 & 0.0 & 13.6 & 6.1 & 67.0 & 31.5 & 23.8 & 11.0 \\
\hline $\mathrm{Cba}$ & 0 & 0 & 37.0 & 22.3 & 0 & 0 & 2.0 & 0.7 & 90.4 & 38.9 & $27.0 \mathrm{ab}$ & $11.8 \mathrm{ab}$ \\
\hline $\mathrm{Ca}$ & 0 & 0 & 41.6 & 26.7 & 0 & 0 & 3.8 & 2.6 & 87.6 & 81.5 & $28.9 \mathrm{ab}$ & $24.1 \mathrm{c}$ \\
\hline $\mathrm{Caq}$ & 0 & 0 & 53.2 & 39.7 & 0 & 0 & 5.0 & 7.9 & 84.8 & 57.7 & $29.8 \mathrm{ab}$ & $22.0 \mathrm{bc}$ \\
\hline $\mathrm{Cb}$ & 0 & 0 & 57.6 & 61.2 & 0 & 0 & 3.2 & 6.8 & 95.0 & 67.1 & $32.5 \mathrm{bc}$ & $27.6 \mathrm{c}$ \\
\hline Су & 0 & 0 & 60.0 & 9.3 & 0 & 0 & 1.2 & 0.0 & 121.2 & 49.5 & $38.0 \mathrm{bc}$ & $12.3 \mathrm{a}$ \\
\hline $\mathrm{Cx}$ & 0 & 0 & 58.6 & 43.9 & 0 & 0 & 1.6 & 6.8 & 144.2 & 79.8 & $42.6 \mathrm{c}$ & $27.2 \mathrm{c}$ \\
\hline Mean $^{\mathrm{z}}$ & 0 & 0 & $45.7 \mathrm{~b}$ & $27.1 \mathrm{~b}$ & 0.0 & 0.0 & $4.1 \mathrm{a}$ & $3.2 \mathrm{a}$ & $95.8 \mathrm{c}$ & $52.8 \mathrm{c}$ & 29.3 & 18.1 \\
\hline $\mathrm{Fe}$ & 0 & 0 & 1.6 & 0.0 & 0 & 0 & 0.2 & 0.6 & 79.2 & 10.8 & 16.9 & $2.5 \mathrm{a}$ \\
\hline $\mathrm{Fa}$ & 0 & 0 & 1.8 & 0.2 & 0 & 0 & 0.4 & 0.0 & 81.8 & 28.2 & 17.5 & $5.9 \mathrm{~b}$ \\
\hline $\mathrm{Fd}$ & 0.2 & 0.0 & 0.4 & 0.0 & 0 & 0 & 0.8 & 0.0 & 92.0 & 42.0 & 19.5 & 8.8 \\
\hline $\mathrm{Fb}$ & 0 & 0 & 0.2 & 0.0 & 0 & 0 & 0.2 & 0.0 & 107.6 & 26.3 & 22.5 & $5.7 \mathrm{~b}$ \\
\hline $\mathrm{Fc}$ & 10.2 & 1.3 & 0.4 & 0.3 & 3.8 & 0.4 & 6.0 & 0.7 & 131.6 & 23.0 & 31.5 & 4.7 \\
\hline Mean $^{z}$ & 0 & 0 & $1.0 \mathrm{a}$ & $0.1 \mathrm{a}$ & 0 & 0 & $1.5 \mathrm{a}$ & $0.2 \mathrm{a}$ & $90.2 \mathrm{~b}$ & $26.8 \mathrm{~b}$ & 19.1 & 5.7 \\
\hline
\end{tabular}

${ }^{\mathrm{w}}$ Contaminated isolates $\mathrm{Ck}, \mathrm{Fc}$, and Fd were excluded from statistical analyses.

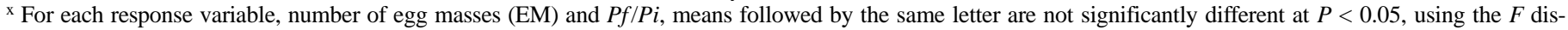
tribution for the deviance statistic on the logit scale for EM and log scale for $P f / P i$.

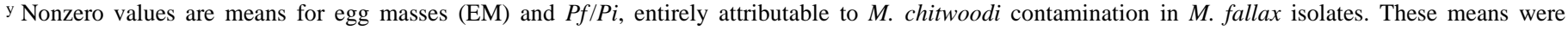
excluded from the marginal means.

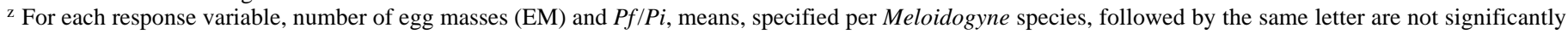
different at $P<0.05$, using the $F$ distribution for the deviance statistic on the logit scale for EM and log scale for $P f / P i$. 
was confirmed by morphological identification of juveniles of $\mathrm{Fc}$, which had reproduced on S. bulbocastanum (G. Karssen, personal communication).

Similarly, morphological identification of the extracted progenies of $\mathrm{Fc}$ from $S$. hougasii and $\mathrm{Fd}$ from $S$. bulbocastanum after harvest indicated that only the contaminating $M$. chitwoodi $\mathrm{J} 2$ in these $M$. fallax isolates were able to reproduce on their respective hosts. Morphological identification also confirmed that the single egg mass produced by $M$. chitwoodi mono-female line $\mathrm{Cb} 1$ on $S$. hougasii (Table 8) was true to species. Species identification by analysis of ITS regions of rDNA revealed the absence of contamination of the isolates with $M$. incognita or other species, which might occur as contaminants under greenhouse conditions. Absolute levels of resistance were demonstrated in S. bulbocastanum and $S$. hougasii genotypes to all tested $M$. fallax and $M$. chitwoodi isolates and mono-female lines, except for contaminated $M$. fallax isolates $\mathrm{Fc}$ and $\mathrm{Fd}$ and mono-female line $\mathrm{Cb} 1$. Because the genotypes of $S$. bulbocastanum and $S$. hougasii showed no differentiation for the two parameters, they did not contribute to the variation and were omitted from the statistical analyses. Consequently, results and discussion of regression analyses are based solely on the genotypes of $S$. chacoense, S. stoloniferum, and $S$. tuberosum.

The interaction terms for isolate-Solanum spp. (Tables 9 and 10) and lines-Solanum spp. (Table 11) were not significant. Plots of deviance residuals against fitted values revealed no anomalies (data not shown). Differences between Solanum spp. were highly significant for both parameters (Tables 9 through 11). Differences between isolates and between mono-female lines of $\mathrm{Cb}$ were significant for both parameters for M. chitwoodi (Tables 9 through 11). For $M$. fallax, differences between isolates were significant for $P f / P i$ but not for number of egg masses. Further, analysis showed a clear Meloidogyne spp.-Solanum spp. interaction effect (data not shown), mainly caused by differences in the responses of $S$. chacoense and S. stoloniferum to the two Meloidogyne species (Table 7).

The M. chitwoodi isolates showed different levels of reproduction on the genotype of $S$. stoloniferum, in contrast to the $M$. fallax isolates, which were avirulent, except for one (Table 7). The differentiating $M$. fallax isolate was the contaminated isolate Fc. The $S$. chacoense genotype varied in susceptibility to $M$. chitwoodi isolates but showed incomplete resistance to $M$. fallax. $\mathrm{Cv}$. Nicola was susceptible to both M. fallax and M. chitwoodi. On this commercial cultivar, the level of susceptibility for the mean number of egg masses was similar for $M$. chitwoodi and $M$. fallax, but the mean $P f / P i$ value was $\approx 50 \%$ lower for $M$. fallax compared to $M$. chitwoodi.

ANOVA of data for root volume revealed no significant differences between $S$. tuberosum, $S$. stoloniferum, and $S$. chacoense (mean root volume values were 2.8, 2.7, and 3.1, respectively; data not shown), suggesting the coparameter root volume had no notable influence on the significant Solanum spp. effect for the parameters.
Using the mean deviance of the residual as an estimate of the dispersion parameter in the logistic and loglinear models resulted in estimates much larger than one (Tables 9 through 11), indicating overdispersion (i.e., the data are from distributions more variable than binomial and Poisson distributions).

\section{DISCUSSION}

Variation in virulence. Considerable variation in virulence was demonstrated among the 14 isolates of M. hapla when tested on the Solanum genotypes. This result agrees with the variation found by Janssen et al. (19). The observed variation was accompanied by a highly significant interaction effect for mean number of egg masses, indicating genetic differences for virulence in $M$. hapla and for resistance versus susceptibility in Solanum genotypes. $M$. hapla is a cytologically complex species, with varying chromosome numbers, ploidy levels, and reproduction systems $(39,40)$. Despite the variability in this species, pathogenic races, as have been described in $M$. chitwoodi, $M$. arenaria, and $M$. incognita, have not yet been reported for M. hapla. Intraspecific variation in $M$. hapla has been recorded twice $(13,14)$, with two M. hapla isolates tested on two genotypes of Medicago sativa. In contrast, the absence of a significant cultivar-isolate interaction effect in white clover was demonstrated when eight $M$. hapla isolates from New Zealand were tested against two resistant and two susceptible cultivars (25). This discrepancy may be due to a possibly narrower genetic basis for M. hapla in New Zealand compared to Western Europe and the United States or to differences in host plants.

The $14 \mathrm{M}$. hapla isolates also were dissimilar. No association was found between virulence and cytological race. Under the assumption that interactions are based on genetic differences, this surprising result indicates the presence of a range of comparable virulence factors in both the meiotic parthenogenetic race $\mathrm{A}$ isolates as well as in the mitotic parthenogenetic race B isolates. Based on the current results and given the random choice of $M$. hapla isolates, there are strong indications that many virulence factors are involved. At least five patterns could be distinguished among the M. hapla isolates based on differences between the numbers of egg masses for each isolate on the three host genotypes (Table 2), implying the presence of approximately five virulence factors in the M. hapla isolates. Apparently, these genes are preserved in $M$. hapla without selection pressure from resistance genes, because these genes have never been introduced before in the Netherlands. We postulate that these virulence factors are maintained in the gene pool by a continuously changing selection pressure, assured by the polyphagous nature and large cytological variation of the nematode species. M. hapla is able to reproduce on many dicotyledons, including weeds. This genetically broad supply of host genotypes may ensure the preservation of many virulence factors.

The absence of significant plant genotype-nematode isolate interactions within $M$. chitwoodi and M. fallax leads us to conclude

TABLE 8. Mean number of egg masses for combinations of Meloidogyne chitwoodi isolate $\mathrm{Cb}$ and mono-female lines of isolate Cb with Solanum bulbocastanum, S. chacoense, S. hougasii, S. stoloniferum, and S. tuberosum

\begin{tabular}{|c|c|c|c|c|c|c|}
\hline \multirow[b]{2}{*}{ Isolate } & \multicolumn{5}{|c|}{ Host } & \multirow[b]{2}{*}{ Isolate mean ${ }^{2}$} \\
\hline & S. bulbocastanum & S. chacoense & S. hougasii & S. stoloniferum & S. tuberosum & \\
\hline $\mathrm{Cb}$ & 0 & 57.8 & 0 & 2.4 & 92.4 & $31.8 \mathrm{bc}$ \\
\hline $\mathrm{Cb} 1$ & 0 & 41.5 & 0.2 & 4.6 & 91.0 & $26.8 \mathrm{a}-\mathrm{c}$ \\
\hline $\mathrm{Cb} 3$ & 0 & 31.0 & 0 & 3.2 & 79.4 & $22.7 \mathrm{a}$ \\
\hline $\mathrm{Cb} 4$ & 0 & 25.0 & 0 & 2.4 & 87.6 & $23.0 \mathrm{a}$ \\
\hline $\mathrm{Cb} 5$ & 0 & 69.2 & 0 & 3.0 & 98.6 & $35.6 \mathrm{c}$ \\
\hline
\end{tabular}

${ }^{\mathrm{z}}$ Means followed by the same letter are not significantly different at $P<0.05$, using the $F$ distribution for the deviance statistic on the logit scale. 
that little variation in virulence exists among the tested isolates of $M$. chitwoodi and M. fallax toward the three Solanum genotypes in this study. These differences in variability in host response between $M$. hapla on one side and M. chitwoodi and M. fallax on the other may be explained by an introduction of $M$. chitwoodi into the Netherlands that is more recent than that of M. hapla. The similarity of the American M. chitwoodi isolate Cba to the Dutch isolates may indicate the same origin or similarity in virulence patterns in $M$. chitwoodi in a wide geographic area. Further studies are needed to provide decisive answers.

Despite the low variation in virulence in relation to Solanum spp., the current study indicates the presence of intraspecific specialization for virulence in $M$. chitwoodi and its absence in the five M. fallax isolates, because the reproduction of isolate Fc on $S$. bulbocastanum and S. hougasii and of $\mathrm{Fd}$ on $S$. bulbocastanum was caused entirely by the presence of $M$. chitwoodi. It is remarkable that the contaminating $M$. chitwoodi in isolate Fc in the current study did not reproduce significantly on $S$. chacoense, as did the nine $M$. chitwoodi isolates (Table 7).

In addition to possible intraspecific specialization, a synergistic effect of the two incompatible Meloidogyne spp. could explain the breaking of $S$. bulbocastanum and $S$. hougasii resistance to $M$. chitwoodi. Synergistic effects inducing susceptibility have been reported in interspecific studies in Meloidogyne but involved inoculation of a compatible Meloidogyne species prior to the incompatible species, such as inoculation with $M$. arenaria or $M$. hapla prior to M. incognita race 1 on tobacco cv. NC95 (11) or M. hapla prior to $M$. incognita race 3 on $M i$-resistant tomato cv. Celebrity (31), using the split-root technique. In an experiment supplementary to the current study, no indication of such a synergistic effect was obtained, because no reproduction was observed with mixtures of $M$. fallax and $M$. chitwoodi isolates as inoculum on $S$. bulbocastanum 93-60-2 (data not shown). To test whether the $M$. chitwoodi contamination in $\mathrm{Fc}$ originated from a host race 3 isolate, this contamination was isolated and tested on S. bulbocastanum 93-60-2 and SB22. No reproduction was observed (data not shown), indicating the $M$. chitwoodi contamination does not belong to host race 3. Moreover, the observations for Fc on $S$. bulbocastanum 93-60-2 in the current paper were not repeatable. Despite the fact that $M$. chitwoodi contaminants did reproduce on $S$. bulbocastanum and $S$. hougasii, we conclude that there is little or no variation in the $M$. chitwoodi isolates.

Differences in variation in virulence can be due either to different rates of virulent and avirulent nematodes within a population, to dosage effects of avirulence genes, or to both. Based on experiments with the mono-female lines, we concluded that genetic variation in pathogenicity is present in the original isolates

TABLE 9. Analyses of deviance for linear regression of egg-mass counts for Meloidogyne chitwoodi and M. fallax isolates inoculated on Solanum chacoense, S. stoloniferum, and S. tuberosum

\begin{tabular}{lrrrc}
\hline Source & df & Deviance & $\begin{array}{c}\text { Mean } \\
\text { deviance }\end{array}$ & $\begin{array}{c}\text { Deviance } \\
\text { ratio }^{z}\end{array}$ \\
\hline M. chitwoodi & & & & \\
$\quad$ Replication & 4 & 157 & 39 & $2.71^{*}$ \\
$\quad$ Isolates & 6 & 257 & 43 & $2.96^{*}$ \\
$\quad$ Solanum spp. & 2 & 4,459 & 2,230 & $154.05^{* * *}$ \\
$\quad$ Residual & 92 & 1,331 & 14 & \\
Total & 104 & 6,204 & 60 & \\
M. fallax & & & & \\
$\quad$ Replication & 4 & 91 & 23 & $3.11^{*}$ \\
Isolates & 2 & 25 & 12 & $1.70 \mathrm{~ns}$ \\
$\quad$ Solanum spp. & 2 & 2,919 & 1,460 & $199.53^{* * *}$ \\
$\quad$ Residual & 36 & 263 & 7 & \\
$\quad$ Total & 44 & 3,298 & 75 & \\
\hline
\end{tabular}

$\mathrm{z} *$ and $* * *$ indicate deviance ratios are significant at $P<0.05$ and $P<0.001$, respectively, and ns indicates not significant, using the $F$ distribution. (e.g., $\mathrm{Cb}$ ), because significant differences exist between these lines. These differences show that a meiotic parthenogenetic $\mathrm{Mel}$ oidogyne population consists of genotypes with different pathogenicity. For breeding purposes, the most pathogenic lines should be chosen, which possibly could be homozygous for virulence gene(s).

Selection for virulence. Several authors have reported selection for virulence under laboratory conditions $(4,20,29,32,38,41)$. In all of these studies, virulent isolates were obtained after several successive generations of selection for virulence originating from avirulent isolates. This laboratory-selected virulence is sometimes confusingly referred to as "B-race" (32). Despite the laboratoryselected virulence of the pathogen, the $M i$-gene in tomato was effective against $M$. incognita, $M$. javanica, and $M$. arenaria from the time of its introgression from Lycopersicon peruvianum to cultivated tomato, until recently (35).

Selection for virulence under natural conditions has been demonstrated, for example, in M. incognita, M. arenaria, and M. javanica on cultivars of groundnut (Arachis hypogaea), cotton (Gossypium hirsutum), strawberry (Fragaria $\times$ ananassa), and sweet pepper (Capsicum frutescens), as discussed by Netscher (30). In the current study, the striking differences between isolates from identical field populations indicates that large genetic variation in virulence can be expected within one field population. Divergence between two isolates from the same field population may be explained by selection of different genotypes during multiplication, beginning with a mixture of genotypes for virulence. Also, contamination of $M$. hapla isolates with other isolates of $M$. hapla cannot be excluded, because no routine detection is currently available to discriminate between isolates of the same species. In contrast, no variation in virulence was found in isolate $\mathrm{Ha}$ when studying five mono-female lines, because no mono-female line-

TABLE 10. Analyses of deviance for loglinear regression of Pf/Pi (final population divided by initial population) of Meloidogyne chitwoodi and $M$. fallax isolates inoculated on genotypes of Solanum chacoense, S. stoloniferum, and S. tuberosum

\begin{tabular}{lrrrr}
\hline Source & df & Deviance & $\begin{array}{c}\text { Mean } \\
\text { deviance }\end{array}$ & $\begin{array}{c}\text { Deviance } \\
\text { ratio }^{\mathrm{z}}\end{array}$ \\
\hline M. chitwoodi & & & & \\
$\quad$ Replication & 4 & 269 & 67 & $6.06^{* * *}$ \\
$\quad$ Isolates & 6 & 497 & 83 & $7.45^{* * *}$ \\
$\quad$ Solanum spp. & 2 & 1,859 & 930 & $83.67^{* * *}$ \\
$\quad$ Residual & 88 & 978 & 11 & \\
$\quad$ Total & 100 & 3,603 & 36 & \\
M. fallax & & & & \\
$\quad$ Replication & 4 & 106 & 26 & $7.04^{* * *}$ \\
Isolates & 2 & 47 & 23 & $6.21^{* * *}$ \\
Solanum spp. & 2 & 631 & 315 & $84.02^{* * *}$ \\
$\quad$ Residual & 34 & 128 & 4 & \\
$\quad$ Total & 44 & 911 & 22 & \\
\hline
\end{tabular}

$\mathrm{z} * * *$ indicates deviance ratio is significant at $P<0.001$, using the $F$ distribution.

TABLE 11. Analysis of deviance for linear logistic regression of egg-mass counts of Meloidogyne chitwoodi isolate $\mathrm{Cb}$ and mono-female lines from isolate $\mathrm{Cb}$ inoculated on Solanum chacoense, S. stoloniferum, and $S$. tuberosum

\begin{tabular}{lrrrr}
\hline Source & df & Deviance & $\begin{array}{c}\text { Mean } \\
\text { deviance }\end{array}$ & $\begin{array}{c}\text { Deviance } \\
\text { ratio }^{\mathrm{z}}\end{array}$ \\
\hline Replication & 4 & 59 & 15 & $2.66^{* *}$ \\
Lines & 6 & 123 & 20 & $3.73^{* *}$ \\
Solanum spp. & 2 & 3,266 & 1,633 & $296.95^{* * *}$ \\
Residual & 86 & 4,73 & 6 & \\
Total & 98 & 3,921 & 40 & \\
\hline
\end{tabular}

$\mathrm{z} * *$ and $* * *$ indicate deviance ratios are significant at $P<0.01$ and $P<$ 0.001 , respectively, using the $F$ distribution. 
Solanum sp. interaction was observed, which indicates the presence of only one common virulence factor. Apparently field populations of $M$. hapla race A may reflect a large diversity in virulence, but once in captivity, a tendency toward homogeneity in virulence can occur due to uniformity in host and environmental conditions. Despite the lack of variation in virulence within isolate Ha, significant differences in mean numbers of egg masses between the mono-female lines were observed. The virulence pattern of Ha was comparable in the two experiments with $M$. hapla isolates, indicating the differences between the lines were caused by genetic differences and were not the result of large environmental effects. The occurrence of more than one virulence factor in a field population should discourage the use of such heterogeneous populations in resistance tests and argues in favor of the use of more homogenous isolates, such as mono-female isolates.

Contamination. For proper testing of isolates, their purity should be guaranteed. One of the factors that influences isolate purity is contamination with nematodes from other Meloidogyne species. Because we used precise methods to test for this kind of contamination, we could identify the few isolates that were contaminated with small numbers of other nematodes. Deviations from consistency in resistance level seemed to be due to contamination, as for isolates $\mathrm{Ck}$ and $\mathrm{Fc}$ on $S$. stoloniferum and isolate $\mathrm{Fc}$ on $S$. bulbocastanum. There are several possible reasons for the contamination of $M$. fallax, varying from mixed field populations to cross-infection during the maintenance program or inoculum preparation. We concluded that despite serious preventive precautions a constant risk for contamination is present in a program using a range of isolates for resistance testing. These mixed-species isolates hinder the interpretation of the results. Only identification of reproduced juveniles, as for isolates $\mathrm{Fc}$ and $\mathrm{Fd}$ on $S$. bulbocastanum and isolate Fc on $S$. hougasii, can limit misinterpretations. These results stress the importance of making every effort to avoid working with contaminated isolates and emphasize the importance of testing for possible contamination. Until now, only contamination with different species could be detected. The degree of genetic homogeneity within a given isolate is unknown.

Resistance. In this paper, M. hapla response varied from total absence to high mean numbers of egg masses on a host genotype. On average, $S$. sparsipilum was significantly more susceptible than the other two Solanum genotypes tested, showing more highly compatible combinations with isolates. The three Solanum genotypes were dissimilar, suggesting differences in genetic control of resistance to the tested isolates.

The significant difference in reproductive ability between $M$. chitwoodi and M. fallax supports the species status of M. fallax, which only recently has been recognized as distinctly different from the related $M$. chitwoodi (22). M. fallax appeared to be less virulent than $M$. chitwoodi on the tested genotypes of $S$. chacoense and $S$. stoloniferum, but both nematode species were equally virulent on $S$. bulbocastanum, S. hougasii, and S. tuberosum cv. Nicola. In general, resistance of Solanum spp. is effective against both $M$. chitwoodi and $M$. fallax, as was shown by Janssen et al. (18) in resistance trials testing 64 Solanum spp.: only a few genotypes of $S$. chacoense and S. stoloniferum (the same genotypes used in this study) were differentiated between these $\mathrm{Mel}$ oidogyne spp. The current study specified the consistency of this differentiation. For practical breeding purposes, however, it is important to use sources for resistance that are effective for both nematode species, because both species are allopatric.

On susceptible $S$. tuberosum cv. Nicola, mean numbers of egg masses were similar for $M$. chitwoodi and $M$. fallax, showing high levels of virulence. However, the mean value of $P f / P i$ for $M$. fallax was $50 \%$ lower than for $M$. chitwoodi. This phenomenon was observed in other experiments as well (44) and was presumed to be due to a shorter life cycle for M. fallax, for which at the time of egg extraction many $\mathrm{J} 2$ had already hatched and left the egg mass. This difference in life cycle was confirmed by M. M. A. Khan (personal communication), who studied the development of these two species in potato and maize. The current results emphasize that when measuring reproduction at the interspecific level, $P f / P i$ values estimated on egg or $\mathrm{J} 2$ counts from extraction with $\mathrm{NaOCl}$ might not be representative when life cycles differ between tested species. Observations of numbers of egg masses, therefore, would be much more reliable than numbers of $P f / P i$ in such situations.

In conclusion, when testing resistance with isolates of $M$. hapla, attention should be paid to the variation in virulence to different Solanum genotypes. Screening for resistance should include testing against a range of isolates that represent the variation in virulence in the species. Furthermore, the efficacy of resistance breeding depends on the durability of the resistance genes. The presence of various genetic factors for virulence amongst $M$. hapla isolates and for resistance in the tested Solanum genotypes stresses the need for genetic analyses. The studied genotypes of $S$. bulbocastanum and $S$. hougasii showed high levels of broad-spectrum resistance to the root-knot nematode species $M$. chitwoodi and $M$. fallax. Additionally, high levels of resistance were demonstrated toward $M$. fallax by the genotypes of $S$. chacoense and $S$. stoloniferum. These results provide promising breeding perspectives. Intraspecific specialization in $M$. chitwoodi and $M$. fallax seems lower and less pronounced and, thus, much easier to handle in breeding programs compared to M. hapla. However, the virulence of $M$. chitwoodi juveniles in isolate Fc on S. bulbocastanum and $S$. hougasii raised overly optimistic expectations because the spread of this and possibly other virulent isolates or races in the Netherlands, as well as the effect of mixtures of species, are not yet known. Because ample knowledge of the durability of resistance in S. bulbocastanum and S. hougasii is still lacking, incomplete resistance like that in $S$. stoloniferum to $M$. chitwoodi should not be discarded.

\section{ACKNOWLEGDMENTS}

This research was supported by EC project FAIR1-CT95-0896. We thank the Plant Protection Service, Wageningen, the Netherlands; the Research Station for Arable Farming and Field Production of Vegetables, Lelystad, the Netherlands; the Hilbrands Laboratory for Soilborne Diseases and Pests, Assen, the Netherlands; the Laboratory for Soil and Crop Testing, Oosterbeek, the Netherlands; Washington State University, Department of Plant Pathology, Prosser; and the Institut National de Recherche Agronomique, Montpellier, France, for providing nematode isolates. We also thank A. van Norel for technical assistance, P. F. G. Vereijken for statistical advice and analyses, and R. F. Hoekstra, J. Hoogendoorn, P. W. Th. Maas, and C. H. van Silfhout for critical reading of the manuscript.

\section{LITERATURE CITED}

1. Austin, S., Pohlman, J. D., Brown, C. R., Mojtahedi, H., Santo, G. S., Douches, D. S., and Helgeson, J. P. 1993. Interspecific somatic hybridization between Solanum tuberosum L. and S. bulbocastanum Dun. as a means of transferring nematode resistance. Am. Potato J. 70:485-495.

2. Baker, R. J., Clarke, M. R. B., and Lane, P. W. 1985. Zero entries in contingency tables. Comput. Stat. Data Anal. 3:33-45.

3. Been, T. H., Meijer, E. M. J., Benier, J. E., and Knol, J. B. 1996. Using image analysis for counting larvae of potato cyst nematodes (Globodera spec.). Fundam. Appl. Nematol. 19:297-304.

4. Bost, S. C., and Triantaphyllou, A. C. 1982. Genetic basis of the epidemiologic effects of resistance to Meloidogyne incognita in the tomato cultivar Small Fry. J. Nematol. 14:540-544.

5. Brown, C. R., Mojtahedi, H., and Santo, G. S. 1989. Comparison of reproductive efficiency of Meloidogyne chitwoodi on Solanum bulbocastanum in soil and in vitro tests. Plant Dis. 73:957-959.

6. Brown, C. R., Mojtahedi, H., and Santo, G. S. 1991. Resistance to Columbia root-knot nematode in Solanum spp. and in hybrids of $S$. hougasii with tetraploid cultivated potato. Am. Potato J. 68:445-452.

7. Brown, C. R., Mojtahedi, H., Santo, G. S., and Austin-Phillips, S. 1994. Enhancing resistance to root-knot nematodes derived from wild Solanum 
species in potato germplasm. Pages 426-438 in: Advances in Potato Pest Biology and Management. G. W. Zehnder, M. L. Powelson, R. K. Jansson, and K. V. Raman, eds. The American Phytopathological Society, St. Paul, MN.

8. Brown, C. R., Yang, C. P., Mojtahedi, H., Santo, G. S., and Masuelli, R. 1996. RFLP analysis of resistance of Columbia root-knot nematode derived from Solanum bulbocastanum in a $\mathrm{BC}_{2}$ population. Theor. Appl. Genet. 92:572-576.

9. Brücher, H. 1967. Root-knot eelworm resistance in some South American tuber-forming Solanum species. Am. Potato J. 44:370-375.

10. Dickson, D. W., and Struble, F. B. 1965. A sieving-staining technique for extraction of egg-masses of Meloidogyne incognita from soil. (Abstr.) Phytopathology 55:497.

11. Eisenback, J. D. 1983. Loss of resistance in tobacco cultivar 'NC95' by infection of M. arenaria or M. hapla. (Abstr.) J. Nematol. 15:478.

12. Esbenshade, P. R., and Triantaphyllou, A. C. 1990. Isozyme phenotypes for the identification of Meloidogyne species. J. Nematol. 22:10-15.

13. Goplen, B. P., Stanford, E. H., and Allen, M. W. 1959. Demonstration of physiological races within three root-knot nematode species attacking alfalfa. Phytopathology 49:653-656.

14. Griffin, G. D., and McKenry, M. V. 1989. Susceptibility of Nevada Synthetic XX germplasm to a California race of Meloidogyne hapla. J. Nematol. 21:292-293.

15. Hoyman, W. G. 1974. Reaction of Solanum tuberosum and Solanum species to Meloidogyne hapla. Am. Potato J. 51:281-286.

16. Hussey, R. S., and Barker, K. R. 1973. A comparison of methods of collecting inocula of Meloidogyne spp. including a new technique. Plant Dis. Rep. 57:1025-1028.

17. Janssen, G. J. W., Van Norel, A., Verkerk-Bakker, B., and Janssen, R. 1995. Detecting resistance to the root-knot nematodes Meloidogyne hapla and $M$. chitwoodi in potato and wild Solanum spp. Potato Res. 38:353-362.

18. Janssen G. J. W., Van Norel, A., Verkerk-Bakker, B., and Janssen, R. 1996. Resistance to Meloidogyne chitwoodi, M. fallax and M. hapla in wild tuber-bearing Solanum spp. Euphytica 92:287-294.

19. Janssen, G. J. W., Van Norel, A., Verkerk-Bakker, B., and Janssen, R. 1997. Intra- and interspecific variation in root-knot nematodes, Meloidogyne spp., for resistance in wild tuber-bearing Solanum species. Fund. Appl. Nematol. 20:449-457.

20. Jarquin-Barberena. H., Dalmasso, A., de Guiran, G., and Cardin, M. C. 1991. Acquired virulence in the plant-parasitic nematode Meloidogyne incognita. 1. Biological analysis of the phenomenon. Rev. Nematol. 14:214-219.

21. Jatala, P., and Bridge, J. 1990. Nematode parasites of root and tuber crops. Pages 137-180 in: Plant Parasitic Nematodes in Subtropical and Tropical Agriculture. M. Luc, R. A. Sikora, and J. Bridge, eds. CAB International, Wallingford, England, U.K.

22. Karssen, G. 1996. Description of Meloidogyne fallax n. sp. (Nematoda: Heteroderidae), a root-knot nematode from the Netherlands. Fundam. Appl. Nematol. 19:593-599.

23. Karssen, G., van Hoenselaar, T., Verkerk-Bakker, B., and Janssen, R. 1995. Species identification of cyst and root-knot nematodes from potato by electrophoresis of individual females. Electrophoresis 16:105-109.

24. McCullagh, P., and Nelder, J. A. 1989. Generalized Linear Models. 2nd ed. Chapman \& Hall, London.

25. Mercer, C. F., and Grant, J. L. 1993. Reproduction of Meloidogyne hapla and Heterodera trifolii from several sites in New Zealand on resistant and susceptible lines of white clover. Nematologica 39:312-321.

26. Mojtahedi, H., Santo, G. S., Brown, C. R., Ferris, H., and Williamson, V. 1994. A new host race of Meloidogyne chitwoodi from California. Plant
Dis. 78:1010.

27. Mojtahedi, H., Santo, G. S., and Wilson, J. H. 1988. Host tests to differentiate Meloidogyne chitwoodi races 1 and 2 and M. hapla. J. Nematol. 20:468-473.

28. Murashige, T., and Skoog, F. 1962. A revised medium for rapid growth and bioassays with tobacco tissue cultures. Physiol. Plant. 15:473-497.

29. Netscher, C. 1977. Observations and preliminary studies on the occurrence of resistance breaking biotypes of Meloidogyne spp. on tomato. Cah. ORSTOM Ser. Biol. 11:173-176.

30. Netscher, C. 1978. Morphological and Physiological Variability of Species of Meloidogyne in West Africa and Implications of Their Control. Veenman, Wageningen, Netherlands.

31. Ogallo, J. L., and McClure, M. A. 1996. Systemic acquired resistance and susceptibility to root-knot nematodes in tomato. Phytopathology 86: 498-501.

32. Riggs, R. D., and Winstead, N. N. 1959. Studies on resistance in tomato to root-knot nematodes and on the occurrence of pathogenic biotypes. Phytopathology 49:716-724.

33. Roberts, P. A. 1992. Current status of the availability, development and use of plant resistance to nematodes. J. Nematol. 24:213-227.

34. Roberts, P. A. 1995. Conceptual and practical aspects of variability in root-knot nematodes related to host plant resistance. Annu. Rev. Phytopathol. 33:199-221.

35. Roberts, P. A., Dalmasso, A., Cap, G. B., and Castagnone-Sereno, P. 1990. Resistance in Lycopersicon peruvianum to isolates of $\mathrm{Mi}$ gene-compatible Meloidogyne populations. J. Nematol. 22:585-589.

36. Santo, G. S., and Pinkerton, J. N. 1985. A second race of Meloidogyne chitwoodi discovered in Washington State. Plant Dis. 69:361.

37. Seinhorst, J. W. 1988. The Estimation of Densities of Nematode Populations in Soil and Plants. Sveriges Lantbruksuniversitet, Uppsala, Sweden.

38. Stephan, Z. A. 1982. The influence of temperature and storage time on eggs of four species of Meloidogyne. Nematol. Mediter. 10:167-173.

39. Triantaphyllou, A. C. 1966. Polyploidy and reproductive patterns in the root-knot nematode Meloidogyne hapla. J. Morphol. 118:403-414.

40. Triantaphyllou, A. C. 1984 . Polyploidy in meiotic parthenogenetic populations of Meloidogyne hapla and a mechanism of conversion to diploidy. Rev. Nematol. 7:65-72.

41. Triantaphyllou, A. C., and Sasser, J. N. 1960. Variation in perineal patterns and host specificity of Meloidogyne incognita. Phytopathology 50: 724-735.

42. Van der Beek, J. G. 1997. Interaction between root-knot nematodes and Solanum spp. Variation in pathogenicity, cytology, proteins and DNA. $\mathrm{Ph} . \mathrm{D}$. thesis. Agricultural University Wageningen, Wageningen, Netherlands.

43. Van der Beek, J. G., Veldhuis, W. B. J., Zijlstra, C., and Van Silfhout, C. H. 1996. Preservation of Meloidogyne hapla and M. chitwoodi in liquid nitrogen: Differences in response between populations. Fundam. Appl. Nematol. 19:227-234.

44. Van der Beek, J. G., Vereijken, P. F. G., Poleij, L. M., and Van Silfhout, C. M. 1998. Isolate-by-cultivar interaction in root-knot nematodes Meloidogyne hapla, M. chitwoodi and M. fallax on potato. Can. J. Bot. 76:75-82.

45. Welham, S. J., and Thompson, R. 1997. Likelyhood ratio tests for fixed model terms using residual maximum likelyhood. J. R. Stat. Soc. B 59:701-714.

46. Zijlstra, C., Lever, A. E. M., Uenk, B. J., and Van Silfhout, C. H. 1995. Differences between ITS regions of isolates of the root-knot nematodes Meloidogyne hapla and M. chitwoodi. Phytopathology 85:1231-1237.

47. Zijlstra, C., Uenk, B. J., and Van Silfhout, C. H. 1997. A reliable, precise method to differentiate species of root-knot nematodes in mixtures on the basis of ITS-RFLPs. Fundam. Appl. Nematol. 20:59-63. 